\title{
New ENDF/B-VII.0 evaluations of neutron cross sections for 32 fission products
}

\author{
Hyeong Il Kim ${ }^{1, a}$, Young-Ouk Lee ${ }^{1}$, Mike Herman ${ }^{2}$, Said F. Mughabghab², Pavel Obložinský ${ }^{2}$, and Dimitri Rochman ${ }^{2}$ \\ ${ }^{1}$ Nuclear Data Evaluation Laboratory, Korea Atomic Energy Research Institute, Daejeon 305-353, Korea \\ ${ }^{2}$ National Nuclear Data Center, Brookhaven National Laboratory, Upton, NY 11973-5000, USA
}

\begin{abstract}
Neutron cross sections for fission products play an important role not only in the design of extended burnup core and fast reactors, but also in the study of the backend fuel cycle and the criticality analysis of spent fuel. New evaluations for the ENDF/B-VII.0 library were performed by the KAERI-BNL collaboration for the following 32 fission products: ${ }^{95} \mathrm{Mo},{ }^{101} \mathrm{Ru},{ }^{103} \mathrm{Rh},{ }^{105} \mathrm{Pd},{ }^{109} \mathrm{Ag},{ }^{131} \mathrm{Xe},{ }^{133} \mathrm{Cs},{ }^{141} \mathrm{Pr}$, and complete isotope chains of ${ }^{142-148,150} \mathrm{Nd}$, ${ }^{144,147,148-154} \mathrm{Sm}$, and ${ }^{156,158,160-164} \mathrm{Dy}$. The evaluations cover a large amount of reaction channels, from $10^{-5} \mathrm{eV}$ to $20 \mathrm{MeV}$, including thermal, resolved, unresolved resonance regions, and fast neutron region.
\end{abstract}

\section{Introduction}

Neutron induced nuclear data for 32 fission products were evaluated under the KAERI-BNL collaboration. All evaluations were adopted by the new US Evaluated Nuclear Data Library, ENDF/B-VII.0, released in December 2006 [1]. The list of fission products consists of priority materials for several applications, and for three isotopic chains, remaining stable isotopes were added to cover the complete list of natural elements. Table 1 shows the full list of fission products evaluated in this work.

Table 1. Fission products evaluated in this work. Shown in bold are priority materials.

\begin{tabular}{ll}
\hline & Nuclides \\
\hline Individual nuclides & ${ }^{95} \mathbf{M o},{ }^{101} \mathbf{R u},{ }^{103} \mathbf{R h},{ }^{105} \mathbf{P d}$, \\
Neodymium & ${ }^{109} \mathbf{A g},{ }^{131} \mathbf{X e},{ }^{133} \mathbf{C s},{ }^{141} \mathbf{P r}$ \\
Samarium & ${ }^{142,143,144,145,146,147,148,150} \mathbf{N d}$ \\
Dysprosium & ${ }^{144,147,148,149,150,151,152,153,154} \mathbf{S m}$ \\
\hline
\end{tabular}

Our evaluation methodology covers both the low energy region and the fast neutron region. In the low energy region, our evaluations are based on the latest data published in the Atlas of Neutron Resonances [2]. This resource was used to infer both the thermal values and the resolved resonance parameters validated against capture resonance integrals. In the unresolved resonance region we used averaged resonance parameters, adjusted to experimental data.

In the fast neutron region, our evaluations are based on the nuclear reaction model code EMPIRE-2.19 [3] validated against the experimental data. EMPIRE is a modular system of codes consisting of many nuclear reaction models, including spherical and deformed optical models, Hauser-Feshbach theory with width fluctuation correction and complete $\gamma$-ray emission cascade, DWBA, Multi-step Direct and Multi-step

\footnotetext{
${ }^{a}$ Presenting author, e-mail: hikim@kaeri.re.kr
}

Compound models, and several versions of the phenomenological preequilibrium models. The code is equipped with a powerful GUI, allowing an easy access to support libraries such as RIPL-2 [4] and CSISRS/EXFOR [5], graphical package, as well utility codes for formatting and checking.

In general, we used the Reference Input Parameter Library, RIPL-2, for the initial set model parameters. These parameters were properly adjusted to reproduce available experimental data taken from the CSISRS/EXFOR library.

\section{Atlas-EMPIRE evaluation methodology}

Our evaluations are based on the Atlas-EMPIRE methodology developed over several recent years by the National Nuclear Data Center, with several external collaborators. This methodology covers both the low-energy region (thermal energy, resolved resonances, unresolved resonances) and the fast neutron energy region. In low-energy region, the evaluations are based on Mughabghab's new Atlas of Neutron Resonances. In the fast-neutron energy region, the evaluations are based on the nuclear reaction model code system EMPIRE developed by Herman et al.

\subsection{Low-energy region}

A statistical analysis of neutron resonances was used by S. Mughabghab, BNL, to produce the well known BNL-325 in the eighties. Its $5^{\text {th }}$ edition was published in 2006 as the "Atlas of Neutron Resonances: Resonance Parameters and Thermal Cross Sections, $\mathrm{Z}=1-100$ " [2], representing a considerable update to the 1981 [6] and 1984 [7] editions of BNL325. These latest thermal values and resonance parameters provided a basis for more than 150 new evaluations included in ENDF/B-VII.0, with resolved and unresolved resonance parameters adopted from ref. [2]. Figure 1 shows the evaluated capture cross sections for ${ }^{133} \mathrm{Cs}$ in thermal and resolved resonance regions compared to available experimental data. 


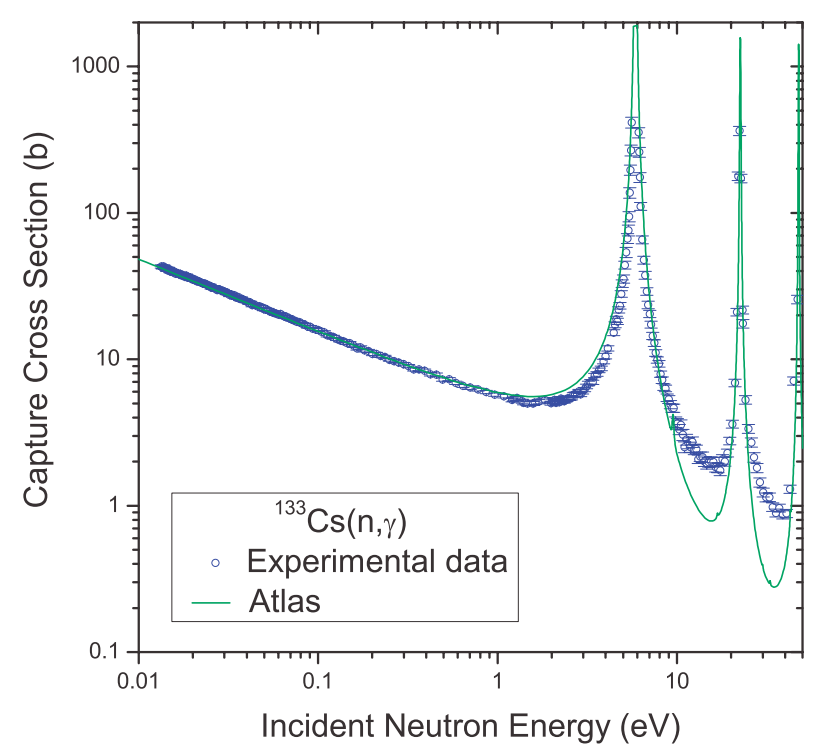

Fig. 1. Neutron capture cross section for ${ }^{133} \mathrm{Cs}$ in the thermal and resolved resonance region up to $50 \mathrm{eV}$.



Fig. 2. Neutron capture cross section for ${ }^{141} \mathrm{Pr}$ in the unresolved resonance region extended up to the first excited level and in the fast neutron region up to $1 \mathrm{MeV}$.

\subsection{Fast neutron region}

All evaluations in the fast neutron region were performed with the new EMPIRE code [3] used for the first time to provide a number of consistent and complete evaluations for a nuclear data library. EMPIRE calculates cross sections for all relevant reaction channels, angular distributions, exclusive and inclusive particle- and $\gamma$-spectra, double-differential cross sections, and spectra of recoils. The code observes angular momentum coupling (at least in the statistical decay part) and is, therefore, capable of detailed modeling of the $\gamma$-cascade providing $\gamma$-ray production spectra, intensities of discrete transitions, and isomeric cross sections.

The evaluation in the fast neutron region was merged with the low energy region at the $1^{\text {st }}$ excited level as shown in figure 2. In order to match these two regions in a smooth way, we usually adjusted the scattering radius in the unresolved
Table 2. Boundary energies between the unresolved resonance region and the fast neutron region for the 32 fission products evaluated in this work.

\begin{tabular}{cc|cc}
\hline Isotopes & $\begin{array}{c}\text { URR limit } \\
\mathrm{keV}\end{array}$ & Isotopes & $\begin{array}{c}\text { URR limit } \\
\mathrm{keV}\end{array}$ \\
\hline${ }^{95} \mathrm{Mo}$ & 206.3 & ${ }^{144} \mathrm{Sm}$ & 540.0 \\
${ }^{101} \mathrm{Ru}$ & 128.4 & ${ }^{147} \mathrm{Sm}$ & 122.05 \\
${ }^{103} \mathrm{Rh}$ & 40.1 & ${ }^{148} \mathrm{Sm}$ & 427.0 \\
${ }^{105} \mathrm{Pd}$ & 283.2 & ${ }^{149} \mathrm{Sm}$ & 22.66 \\
${ }^{109} \mathrm{Ag}$ & 88.8 & ${ }^{150} \mathrm{Sm}$ & 336.11 \\
${ }^{131} \mathrm{Xe}$ & 80.8 & ${ }^{151} \mathrm{Sm}$ & 66.26 \\
${ }^{133} \mathrm{Cs}$ & 81.6 & ${ }^{152} \mathrm{Sm}$ & 122.59 \\
${ }^{141} \mathrm{Pr}$ & 146.5 & ${ }^{153} \mathrm{Sm}$ & 6.5 \\
${ }^{142} \mathrm{Nd}$ & 200.0 & ${ }^{154} \mathrm{Sm}$ & 82.52 \\
${ }^{143} \mathrm{Nd}$ & 225.0 & ${ }^{156} \mathrm{Dy}$ & 138.72 \\
${ }^{144} \mathrm{Nd}$ & 250.0 & ${ }^{158} \mathrm{Dy}$ & 99.55 \\
${ }^{145} \mathrm{Nd}$ & 67.69 & ${ }^{160} \mathrm{Dy}$ & 87.34 \\
${ }^{146} \mathrm{Nd}$ & 456.97 & ${ }^{161} \mathrm{Dy}$ & 25.81 \\
${ }^{147} \mathrm{Nd}$ & 50.3 & ${ }^{162} \mathrm{Dy}$ & 81.16 \\
${ }^{148} \mathrm{Nd}$ & 300.0 & ${ }^{163} \mathrm{Dy}$ & 73.90 \\
${ }^{150} \mathrm{Nd}$ & 130.97 & ${ }^{164} \mathrm{Dy}$ & 73.84 \\
\hline
\end{tabular}

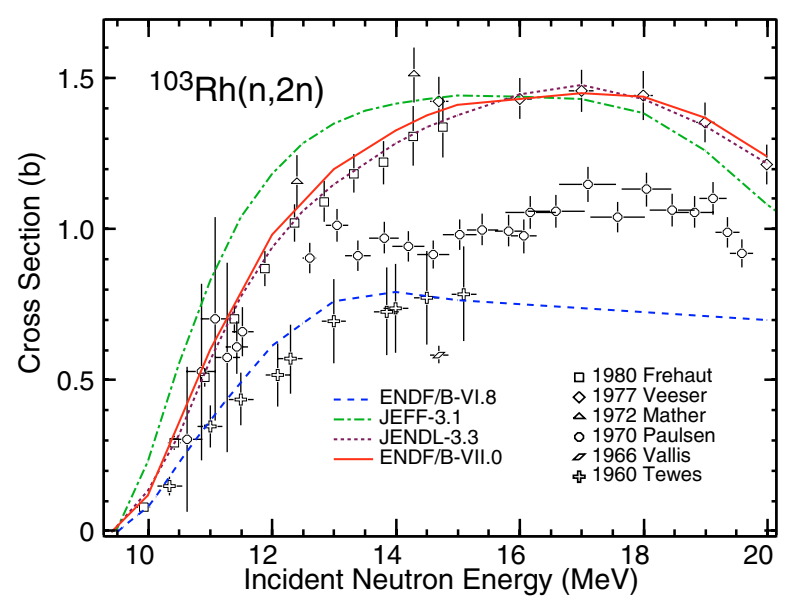

Fig. 3. ENDF/B-VII.0 evaluated (n, 2n) cross section for ${ }^{103} \mathrm{Rh}$ compared to the ENDF/B-VI.8, JEFF-3.1 and JENDL-3.3 evaluations and to the measurements.

region, $R^{\prime}$, and inserted negative cross sections background on rare occasions. Table 2 shows boundary energies between the unresolved resonance region and the fast neutron region.

\subsection{Evaluation of complete isotopic chains}

A new feature of our evaluation work is a simultaneous evaluation of a complete isotopic chain for a given element. This is nowadays possible with the EMPIRE package, which takes advantage of the tremendous progress in the development of evaluation tools in recent years. The combined capabilities of the Atlas of Neutron Resonances [2], the nuclear reaction model code EMPIRE [3], input parameter libraries such as the Reference Input Parameter Library, RIPL-2 [4], experimental cross section library CSISRS/EXFOR [5], and numerous ENDF formatting and checking utilities has facilitated such a complex evaluation work. A considerable advantage of this approach is a full and consistent utilization of data that are 


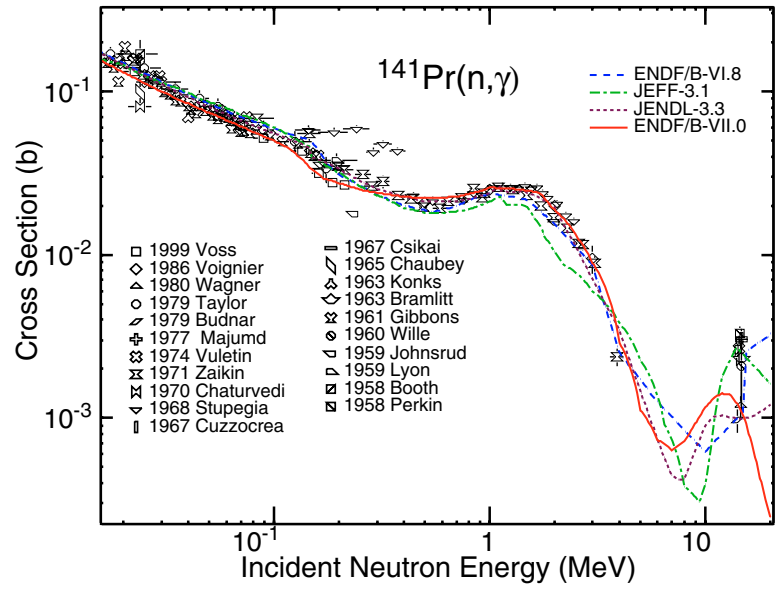

Fig. 4. ENDF/B-VII.0 evaluated $(\mathrm{n}, \gamma)$ cross section for ${ }^{141} \mathrm{Pr}$ in the fast neutron energy region compared to the ENDF/B-VI.8, JEFF-3.1 and JENDL-3.3 evaluations and to the measurements.

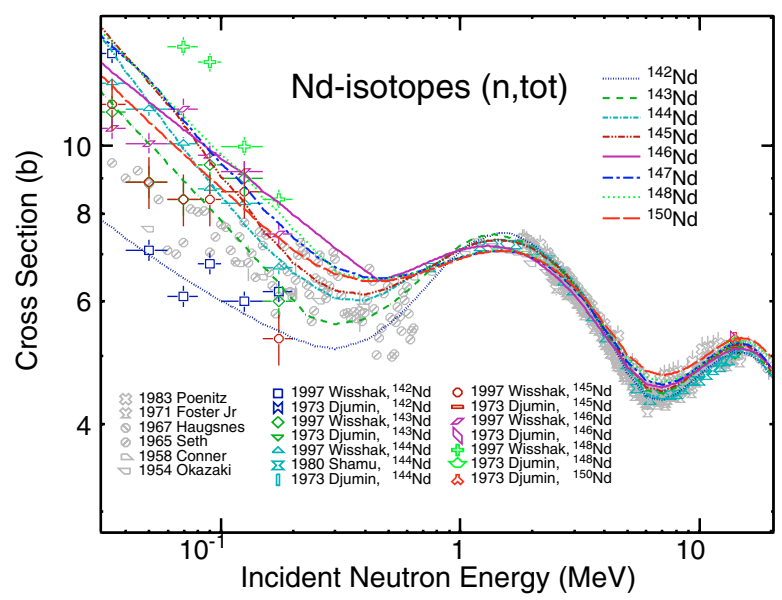

Fig. 5. Total cross sections for all neodymium isotopes compared to measurements.

often measured on natural elements rather than isotopes, a consistent application of model parameters, and comparison with data by summing up isotopic evaluations into a single elemental representation.

\section{Results}

In the following, we compared our new evaluations to the most recent major data libraries, JEFF-3.1 (released in 2005), JENDL-3.3 (released in 2002) and ENDF/B-VI.8 (released in 2001), and to available experimental data. Figure 3 presents a comparison of the ${ }^{103} \mathrm{Rh}(\mathrm{n}, 2 \mathrm{n})$ evaluations with experimental data. Our evaluation reproduces the newer experimental data $[8,9]$ well, which are higher than the older ones [10, 11]. We note that ENDF/B-VI.8 is lower than other libraries and experimental data, and it shows unphysical behavior at high energies, due to the emission of the $(n, 3 n)$ reaction channel. Capture cross sections for ${ }^{141} \mathrm{Pr}$ are compared to recent libraries and measurements as shown in fall 4 . Our results reproduce the experimental data [12-18] below and above the URR.

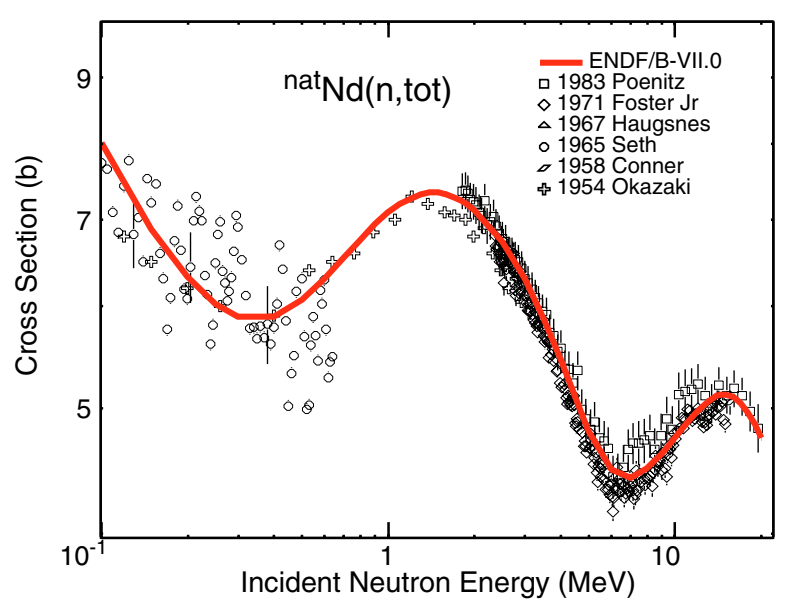

Fig. 6. ENDF/B-VII.0 total cross section for ${ }^{\text {nat }} \mathrm{Nd}$ compared to measurements for natural $\mathrm{Nd}$.

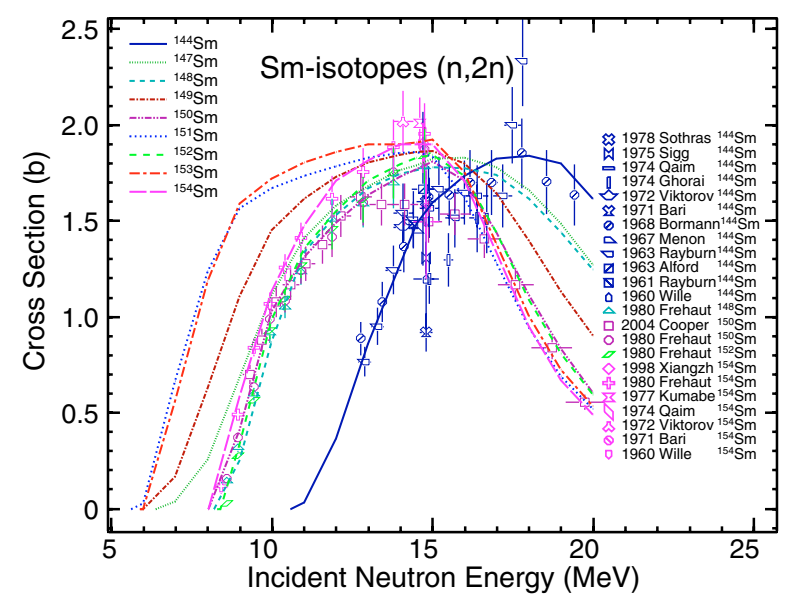

Fig. 7. ENDF/B-VII.0 (n,2n) cross sections for all samarium isotopes compared to measurements.

For three isotopic chains (Nd, Sm, and Dy), we illustrate advantages of the simultaneous evaluation by plotting together ENDF/B-VII.0 evaluated cross sections for the major reaction channels. Figure 5 presents the ENDF/B-VII.0 total cross sections for all neodymium isotopes compared with the measured data. In general, our evaluations agree well with experimental data. The only exceptions are the two points by Wisshak for ${ }^{148} \mathrm{Nd}$ which appear to be suspiciously high. Our results are also in good agreement with the measured total cross section on ${ }^{\text {nat }} \mathrm{Nd}$ as shown in figure 6 . In this case, the calculated cross sections for natural element were obtained as a sum of cross sections for all individual isotopes weighted by their respective abundances. Figure 7 shows a full set of $(n, 2 n)$ cross sections for all samarium isotopes. When experimental data exist for the ${ }^{144,148,150,152,154} \mathrm{Sm}$ isotopes, our evaluations reproduce them well. Figure 8 shows the ENDF/B-VII.0 capture cross sections of all dysprosium isotopes compared to experimental data.

\section{Conclusions}

Neutron cross sections for 32 fission products were evaluated from $10^{-5} \mathrm{eV}$ to $20 \mathrm{MeV}$ under the KAERI-BNL collaboration 


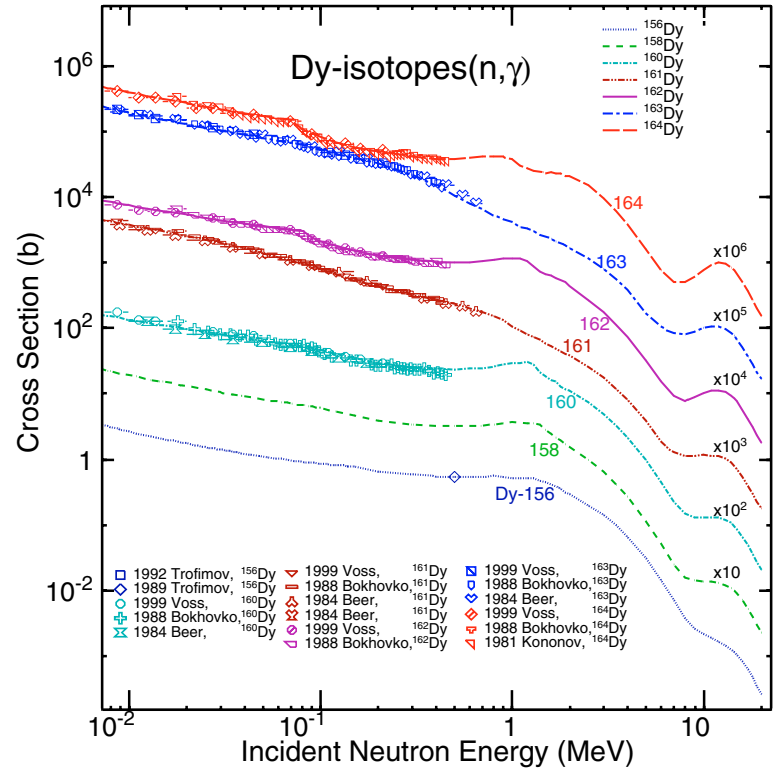

Fig. 8. ENDF/B-VII.0 capture cross sections for all dysprosium isotopes compared to measurements.

for the US ENDF/B-VII.0 library, released in December 2006. The list of fission products consists of the priority materials for several applications, extended to cover complete isotopic chains for several elements. The full list includes 8 individual isotopes, ${ }^{95} \mathrm{Mo},{ }^{101} \mathrm{Ru},{ }^{103} \mathrm{Rh},{ }^{105} \mathrm{Pd},{ }^{109} \mathrm{Ag},{ }^{131} \mathrm{Xe},{ }^{133} \mathrm{Cs}$, ${ }^{141} \mathrm{Pr}$, and 24 isotopes in complete isotopic chains for $\mathrm{Nd}(8)$, Sm (9) and Dy (7). The evaluated data were converted into ENDF-6 formatted files, verified with a set of checking codes, processed with the code NJOY-99.161, and subjected to test runs with the code MCNP5 to ensure that the files can be used in transport calculations.

This work has been performed under the auspices of the Korean Ministry of Science and Technology as the long-term R\&D project.

\section{References}

1. M. Chadwick, P. Obložinský, M. Herman et al., Nucl. Data Sheets 107(12), 2931 (2006).

2. S.F. Mughabghab, Atlas of Neutron Resonances: Thermal Cross Sections and Resonance Parameters (Elsevier, Amsterdam, 2006).

3. M. Herman, R. Capote, B. Carlson, P. Obložinský, M. Sin, A. Trkov, V. Zerkin, EMPIRE nuclear reaction model code, version 2.19 (Lodi), www.nndc.bnl.gov/empire219/ (2005).

4. P. Young, M. Herman, P. Obložinský, T. Belgya, O. Bersillon, R. Capote, T. Fukahori, G. Zhigang, S. Goriely, A. Ignatyuk et al., TECDOC-1506, IAEA, Vienna (2006).

5. NRDC-Network, CSISRS/EXFOR library of experimental cross sections, www.nndc.bnl.gov/exfor (2006).

6. S.F. Mughabghab, M. Divadeenam, N.E. Holden, Neutron Cross Sections: $Z=1-60$, Vol. 1A (Academic Press, New York, 1981)

7. S.F. Mughabghab, Neutron Cross Sections: $Z=61-100$, Vol. 1B (Academic Press, New York, 1984).

8. L.R. Veeser, E.D. Arthur, P.G. Young, Phys. Rev. C 16, 1792 (1977).

9. J. Frehaut, A. Bertin, R. Bois, J. Jary (1980) (private communication).

10. D.G. Vallis, Tech. Rep. 76/66, A.W.R.E. Aldermaston (1966)

11. H.A. Tewes, A.A. Caretto, A.E. Miller, D.R. Nethaway, Tech. Rep. 6028, U.C., Lawrence Rad. Lab. (Berkeley and Livermore) (1960).

12. F. Voss, K. Wisshak, C. Arlandini, F. Kaeppeler, L. Kazakov, T. Rauscher, Phys. Rev. C 59(2), 1154 (1999).

13. R.B. Taylor, B.J. Allen, A.R.D. Musgrove, R.L. Macklin, Australian J. Phys. 32, 551 (1979).

14. J.H. Gibbons, R.L. Macklin, P.D. Miller, J.H. Neiler, Phys. Rev. 122, 182 (1961).

15. J. Voignier, S. Joly, G. Grenier, Nucl. Sci. Eng. 93, 43 (1986).

16. D.C. Stupegia, M. Schmidt, C.R. Keedy, A.A. Madson, J. Nucl. Energy 22, 267 (1968).

17. G.G. Zaikin, I.A. Korzh, M.V. Pasechnic, N.T. Skljar, Ukrainskii Fizichnii Zhurnal 16(7), 1205 (1971).

18. M. Wagner, H. Warhanek, Acta Physica Austriaca 52, 23 (1980). 